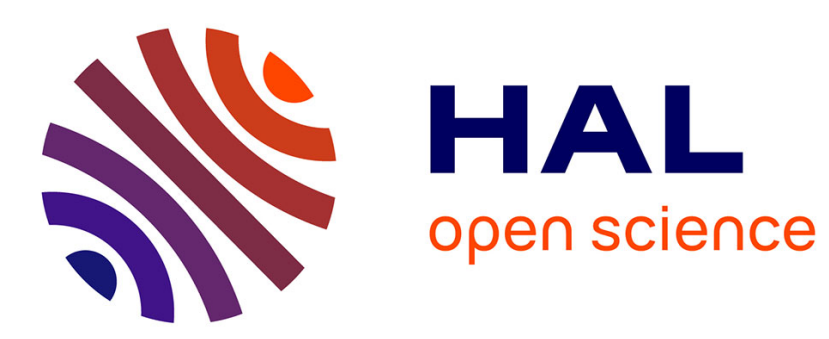

\title{
Coordination, subordination, deixis et focalisation en arabe de Yafi' (Yémen)
}

Martine Vanhove

\section{To cite this version:}

Martine Vanhove. Coordination, subordination, deixis et focalisation en arabe de Yafi' (Yémen).

Oriente Moderno, 2000, XIX (LXXX) (n.s., 1), pp.209-223. halshs-00009712

\section{HAL Id: halshs-00009712 \\ https://shs.hal.science/halshs-00009712}

Submitted on 22 Mar 2006

HAL is a multi-disciplinary open access archive for the deposit and dissemination of scientific research documents, whether they are published or not. The documents may come from teaching and research institutions in France or abroad, or from public or private research centers.
L'archive ouverte pluridisciplinaire HAL, est destinée au dépôt et à la diffusion de documents scientifiques de niveau recherche, publiés ou non, émanant des établissements d'enseignement et de recherche français ou étrangers, des laboratoires publics ou privés. 


\title{
COORDINATION, SUBORDINATION, DEIXIS ET FOCALISATION EN ARABE DE YAFI‘ (YEMEN)
}

\author{
Martine Vanhove \\ CNRS-LLACAN \\ 4ter, route des Gardes \\ 92190 MEUDON - FRANCE
}

\section{Introduction}

La région de Yafi‘, située à environ deux cents kilomètres au nord-est d'Aden en territoire montagneux, représente l'extrême limite méridionale des "dialectes en -k » qui s'étendent du nord au sud dans la vaste zone des hauts plateaux yéménites. Il s'agit de dialectes dont la caractéristique commune la plus saillante est l'utilisation comme suffixe d'accompli d'un morphème à base -k- (comme dans les langues sudarabiques modernes et les langues éthio-sémitiques) au lieu de -t-. Ceux de la région de Yafi“ se subdivisent en quatre grands sous-groupes (Vanhove 1995).

L'objet de cet article est d'étudier le fonctionnement de la particule wun, bien connue sous des formes plus ou moins agglutinées en arabe: wa ?an(na), wa ?in(na), $>$ win, winn. Si les dialectes de Yafi“ partagent avec d'autres variétés d'arabe les valeurs qui lui sont généralement attribuées de présentatif et d'expression de la conséquence, ils n'en présentent pas moins un certain nombre de particularités syntaxiques, sémantiques et énonciatives qui méritent une étude détaillée. Elles conduiront à s'interroger sur la nécessité de classer ce morphème au sein d'une catégorie grammaticale plutôt qu'une autre, à savoir conjonction de coordination, de subordination ou présentatif, et à en proposer, pour les dialectes de cette région, une interprétation en terme de focalisation.

Le travail repose exclusivement sur les 66 énoncés extraits d'un corpus de quarante-deux contes, facéties et légendes hagiographiques recueillis sur le terrain entre 1994 et $1998 .{ }^{1}$ En effet, ce type de récits, comme les récits d'événements marquants survenus aux locuteurs ou à leur entourage, ${ }^{2}$ se prête bien à l'utilisation de telles constructions. On notera cependant une disparité dans la fréquence d'emploi entre, d'une part, la locutrice d'Ar-Rubbi'i, et, d'autre part, tous les autres locuteurs :

$\begin{array}{llll}\text { locutrice de Ar-Rubbi'i } & 59 / 3000^{3} & \text { soit } & 1,97 \% \\ \text { locutrice de Mash'ala } & 4 / 534 & \text { soit } & 0,75 \% \\ \text { locutrice de Ben Salem } & 1 / 113 & \text { soit } & 0,88 \% \\ \text { locuteur de Rusud } & 1 / 166 & \text { soit } & 0,60 \% \\ \text { autre locutrice de Mash'ala } & 0 / 523 & \text { soit } & 0 \% \\ \text { locutrice de Wadi ad-Daxla } & 0 / 222 & \text { soit } & 0 \% \\ \text { locuteur de Shemsan } & 0 / 97 & \text { soit } & 0 \%\end{array}$

\footnotetext{
${ }^{1}$ Mes informateurs sont cinq locutrices âgées de 20 à 55 ans appartenant à des communautés dialectales différentes : Ar-Rubbi ‘i, Mash'ala (M), Wadi ad-Daxla (W) et Ben Salem (B). Seulement deux des contes m'ont été racontés par des hommes âgés originaires d'autres communautés : Rusud (R) et Shemsan. Sauf mention contraire, les exemples proviennent de la locutrice d'Ar-Rubbi'i auprès de laquelle j'ai enregistré le plus de récits (29).

${ }^{2}$ Comme dans le récit du drame de Sham'ah (Landberg 1905 : 7-14) qui relate les combats qui ont longtemps opposé les tribus du Datînah, et à l'inverse des textes descriptifs qui n'y ont pas recours : ainsi, aucun exemple n'a été relevé dans les descriptions de métiers traditionnels ou de coutumes diverses dans Landberg (1901 et 1905).

${ }^{3}$ Le premier chiffre renvoie au nombre d'occurrences de wun, le second au nombre d'énoncés produits par l'informateur.
} 
Même ramenée à un nombre de phrases équivalent dans des textes pris au hasard, l'utilisation que la locutrice d'Ar-Rubbi'i fait de cette particule est toujours plus de deux fois supérieure à celle des autres locuteurs. Les raisons de cet écart peuvent être multiples et ne sont pas mutuellement exclusives : des différences idiolectales, dialectales, sociolectales entre les classes d'âge et les sexes, le degré de contact avec les variétés prestigieuses ou encore éventuellement la nature des sujets traités. Etant donnée l'inégalité quantitative des données recueillies auprès des différents locuteurs (les hommes sont particulièrement peu représentés), il convient de rester prudent quant aux conclusions à tirer, et l'on constatera simplement que la plus prolixe en ce qui concerne l'usage de wun est une femme, âgée de 55 ans, illettrée, originaire et résidant à Ar-Rubbi'i et n'ayant que très peu quitté son hameau natal. Ceux qui en ont fait le moins usage sont des hommes, des femmes instruites, ayant voyagé, jeunes pour trois sur quatre d'entre elles, et tous originaires d'autres régions de Yafi'.

\section{La double origine de wun}

Les grammaires de l'arabe classique soulignent toutes le fonctionnement multiple de certaines conjonctions de subordination et de coordination. Ainsi Blachère et GaudefroyDemombynes (1975: 212-4, 380-1, 436-441, 443) notent-ils que ?in(na) ou ?an(na) introduisent des subordonnées, des complétives, marquent une constatation, une conséquence et sont aussi des "expositifs". ${ }^{4}$ Fernandez (1994:100) souligne en outre sa fonction thématisante. Parallèlement, wa, tout en étant classé comme conjonction de coordination, se «trouve cependant dans certains cas en tête de subordonnées ... pour marquer que la subordonnée exprime un procès concomitant de celui énoncé en principal » (Blachère et Gaudefroy-Demombynes 1975: 443-4). Watson (1993: 281, 425) rend compte de phénomènes similaires pour l'arabe de Sanaa.

La réunion de ces deux particules dans nombre de dialectes arabes, dont ceux du Yémen, permet l'expression d'un présentatif comme pour l'arabe de Sanaa (Watson $1993: 425$ ) ou du Datînah (il est dénommé «affirmatif» par Landberg (1905: 351) qui juge inutile de le traduire). Les traductions proposées par Piamenta (1990-1991: 14) apportent quelques précisions : w-in est rendu par « and then, all of a sudden » et $\mathbf{w}-\mathbf{i n n}$ par « and I found out that ; and behold, and lo, all of a sudden, suddenly », traductions qui, au-delà du présentatif, expriment la consécution et une idée de soudaineté, d'inattendu.

Pour décrire les combinaisons syntaxiques tolérées par ces morphèmes, nous sommes tributaires des descriptions connues. Les quelques exemples relevés dans Rossi, Watson et Piamenta ne donnent que des propositions nominales après w-in(n). Quant à l'arabe du Datînah, s'il ne tolère qu'un nom ou un pronom personnel directement après $\mathbf{w}$-in(n), il relie aussi bien des propositions nominales que verbales.

A Yafi', dans la plupart des cas (cf. § 3), les deux particules présentent des valeurs en partie similaires et sont totalement agglutinées en une forme wun (variantes un, rarement wen). On relève encore cependant quelques exemples où celles-ci sont encore nettement distinctes et peuvent même être séparées par un déictique. Elles sont alors toutes suivies de propositions nominales, mais ceci peut être le fruit du hasard étant donné le petit nombre d'occurrences relevées :

(le mari vérifie le contenu du sac rempli par sa femme)

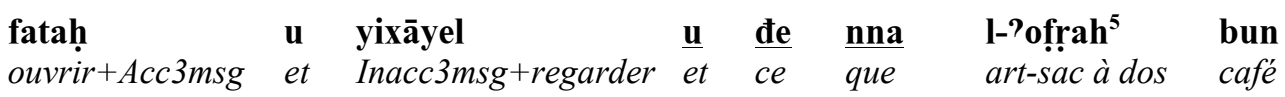

Il a ouvert, il a regardé, et c'était bien un sac de café.

$$
\text { daxal }
$$

wu la

u inna s-sāhạeb rōs

el-mitka?ah

\footnotetext{
${ }^{4}$ C'est-à-dire des présentatifs. Il est précisé (p. 213 et 381) que les particules équivalent aux deux points (:) de la graphie.

${ }^{5}$ La fricative vélaire sonore $\dot{\mathbf{g}}$ passe souvent à la laryngale $?$ ou à l'uvulaire q. Dans le premier cas cela entraine une vélarisation de la ou des consonnes suivantes (phénomène co-articulatoire qui la distingue de la laryngale étymologique).
} 
entrer+Acc3msg et voilà et que art-ami sur art-coussin

Il est entré et voilà que son amant était accoudé sur un coussin.

(Un homme en assure un autre de son amitié, d'où la surprise de ce dernier lorsqu'il constate qu'il le charge de lourds cailloux, signe d'amitié plutôt douteux)
hamelū-hā
wu in
el-masab
ma'a-h mushet-oh
ḥigār
charger+Acc3mpl-elle et que art-sac àdos avec-lui contenu de-lui pierres

Ils la chargeaient et voilà que le sac à dos qu'il avait contenait des pierres.

On notera que lorsque Pin(na), employé seul, sans wu, introduit des complétives, il peut être directement suivi d'un verbe, comme dans les autres variétés d'arabe :
?ána ?asál-ak
in yigî́ōn
l-ī $\quad$ awécleh
je Inacclsg+demander-toi $+m$ que Inacc3mpl+venir à-moi enfants

Moi, je te demande de me donner des enfants.

De plus, ?inna (variante anna, souvent réduit à nna) et ?in, employés seuls, permettent aussi de former diverses conjonctions de subordination composées à valeur temporelle, finale, etc. (ex. 5) et peuvent fonctionner comme des présentatifs (ex. 6) :

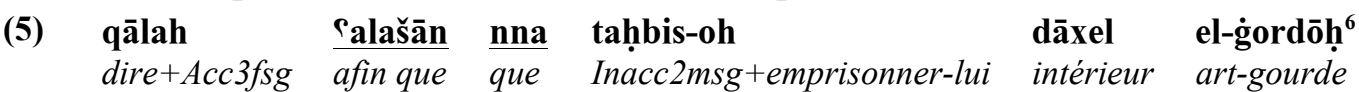

Elle a dit : c'est pour que tu l'emprisonnes à l'intérieur de la gourde.

(6)

$\begin{array}{llllll}\text { anna } & \begin{array}{l}\text { n-nafs } \\ \text { que }\end{array} & \text { bi } & \text { n-nafs, } & \text { ?ámar } & \text { aḷáh } \\ \text { avec } & \text { art-même } & \text { ordonner+Acc3msg } & \text { Dieu }\end{array}$

Voilà, donnant donnant, a ordonné Dieu.

Quant à wu, non suivie de Pin(na), il fonctionne comme la conjonction de coordination de l'arabe classique et peut relier aussi bien des suites de mots que des suites de propositions.

En résumé, la particule wun de Yafi ' provient bien de l'agglutination de deux particules, l'une, wu, habituellement classée comme conjonction de coordination, mais avec des emplois qui la rapprochent des conjonctions de subordination, l'autre, ?in(na), fonctionnant à la fois comme une conjonction de subordination et comme un présentatif, diachroniquement comme synchroniquement.

\section{Le fonctionnement syntaxique de wun}

A Yafi', l'utilisation de wun est soumise à certaines contraintes syntaxiques : il ne peut relier que des propositions, nominales ou verbales (quelle que soit la conjugaison), jamais des suites de mots (noms, verbes ou autres) :

\begin{tabular}{|c|c|c|c|c|c|c|c|c|}
\hline $\begin{array}{l}\text { điyah } \\
c e\end{array}$ & $\begin{array}{l}\text { bena-hā } \\
\text { fils-elle }\end{array}$ & $\begin{array}{l}\text { qurdūh } \\
\text { gourde }\end{array}$ & $\begin{array}{l}\text { laban } \\
\text { lait }\end{array}$ & $\begin{array}{l}\text { ma } \\
\text { quoi }\end{array}$ & $\begin{array}{l}\mathbf{C a}^{7} \\
\text { encore }\end{array}$ & $\begin{array}{l}\text { lla } \\
\text { sauf }\end{array}$ & $\begin{array}{l}\text { l-ah } \\
\grave{a}-l u i\end{array}$ & $\begin{array}{l}\text { l-qur'eh, } \\
\text { art-bord de couvercle }\end{array}$ \\
\hline $\begin{array}{ll}\frac{\text { wun }}{e t^{8}} & \text { đa } \\
c e\end{array}$ & $\begin{array}{l}\text { hasīn } \\
\text { joli }\end{array}$ & $\begin{array}{l}\text { maxḍab } \\
\text { gourde }\end{array}$ & $\begin{array}{l}\text { li } \\
\grave{a}\end{array}$ & & & & & \\
\hline
\end{tabular}

Et celle-ci, son fils était bete comme une gourde de lait dont il ne reste que le bord du couvercle en paille. Et c'est qu'il était vraiment une belle gourde à lait ! (= il était complètement idiot)

(8)

$\begin{array}{llllll}\begin{array}{l}\text { wúșuleh } \\ \text { arriver+Acc3fsg }\end{array} & \text { un } & \begin{array}{l}\text { s-șábī } \\ \text { et }\end{array} & \begin{array}{l}\mathbf{q a}^{\mathbf{9}} \\ \text { art-garçon }\end{array} & \mathbf{h} \overline{\mathbf{o}} & \mathbf{q u ́ r r a ̄ n i} \\ \text { déjà } & \text { il } & \text { raide mort }\end{array}$

\footnotetext{
${ }^{6}$ Les dialectes de Yafi“ se caractérisent par une liberté de l'articulation de l'uvulaire qui varie de $\mathbf{q}$ à $\dot{\mathbf{g}}$ en passant par $\mathbf{6}$.

${ }^{7}$ Forme abrégée de $\Upsilon^{\mathbf{a} d}$.

${ }^{8}$ La particule wun a été glosée systématiquement par "et", sens du premier élément qui la compose. Ceci ne doit pas être compris comme une prise de position quant à sa valeur, mais comme une simple commodité avant d'en avoir démontré le fonctionnement réel.

${ }^{9}$ Forme abrégée de qad.
} 
Elle arriva mais c'est que le garçon était déjà raide mort !

(9)
u hu gerr
el-fás
u yídmax
el-ġașếṣ, wen đa falấsīs
et il prendre+Acc3msg art-hache et Inacc3msg+casser art-marmite et ce miettes

Et lui, il a attrapé la hache et il a cassé la marmite et la voilà en miettes !

(10)

$\begin{array}{llllll}\text { trákkađ̣ } & \text { u } & \text { trákkađ̣ } & \text { wun } & \text { đāa } & \text { māt } \\ \text { ruer+Acc3msg } & \text { et } & \text { ruer+Acc } 3 m s g & \text { et } & \text { ce } & \text { mourir }+A c c 3 m s g\end{array}$

Il a rué, et rué et voilà qu'il est mort.

(11) riga

un đa șot-hā bi-yirtefe ${ }^{\mathrm{a}}$

revenir+Acc3msg et ce voix-elle conc-Inacc3msg+être monté

Il est revenu et voilà que sa voix montait.

Wun, en dehors de son origine, est aussi, sur le plan syntaxique, fortement lié à la deixis puisque dans 59 occurrences sur 66, il est au minimum suivi d'un pronom démonstratif (voir les ex. 7 et 9-11 ci-dessus). Dans 17 cas, il est même aussi précédé d'un autre présentatif du dialecte, la, illa « voilà ». Celui-ci a aussi été relevé dans deux autres exemples où ne figure aucun pronom démonstratif. Restent donc seulement 5 exemples où aucun élément déictique fort $^{10} \mathrm{n}$ 'apparaît. Ce recours quasi systématique à des déictiques semble être une spécificité propre aux dialectes de Yafi', puisque, à en juger par les exemples relevés au Yémen chez Landberg, Rossi (1939) et Watson, ni ceux du Datînah, ni celui de Sanaa n'en font usage :

(12)

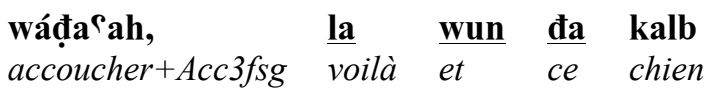

Elle a accouché et voilà que c'était un chien.

\begin{tabular}{|c|c|c|c|c|c|}
\hline $\begin{array}{l}\text { šāf } \\
\text { voir }+A c c 3 m s g\end{array}$ & $\begin{array}{ll}\text { la, } & \mathbf{w} \\
\text { vers } & \text { et }\end{array}$ & $\begin{array}{l}\text { el-?ubắár } \\
\text { art-poussière }\end{array}$ & $\begin{array}{l}\text { bi-yíț!a' }{ }^{\top}, \\
\text { conc-Inacc } 3 m s g+\text { monter }\end{array}$ & $\begin{array}{l}\text { hấđa } \\
c e\end{array}$ & $\begin{array}{l}\text { l-wúled, } \\
\text { art-enfant }\end{array}$ \\
\hline $\begin{array}{l}\text { el-9ubọá } \\
\text { poussièr }\end{array}$ & $\begin{array}{ll}\mathbf{r} & \text { bi-yítb } \\
e & \text { conc-In }\end{array}$ & acc3msg+suivre & & & \\
\hline
\end{tabular}

Il a vu, ce garçon, et la poussière montait, et c'est que la poussière suivait.

Du point de vue de la deixis, on remarquera que la construction avec wun est à plusieurs reprises mise en parallèle syntaxiquement avec des structures comportant aussi des éléments déictiques, à savoir des présentatifs (?ala, ma, ta, ?an) :
?ála mā hū hadd,
gálas
un đē mấšī
voilà quoi il selever+Acc3msg rester+Acc3msg et ce nég

Et voilà que son fils ne bougeait pas, et c'est qu'il ne disait ${ }^{11}$ rien.

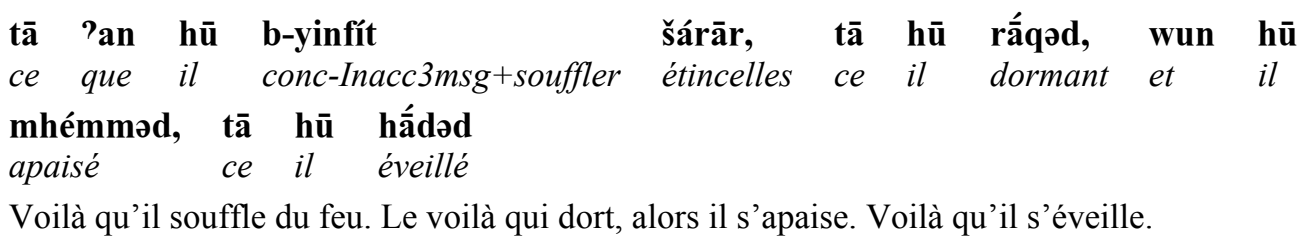

En dehors des démonstratifs, le plus souvent invariables et figés au masculin singulier, wun peut être suivi, comme dans d'autres dialectes du Yémen, d'un nom (3 occurrences) ou d'un pronom sujet indépendant (2 occurrences). Il n'est qu'une seule fois directement suivi d'un verbe. Cette exception pourrait s'expliquer de trois manières: il s'agit soit d'une variante sub-dialectale, soit d'une évolution en cours représentée dans le parler des jeunes générations (la locutrice est âgée d'une vingtaine d'années) qui ne serait pas attestée chez

\footnotetext{
${ }^{10}$ On entend ici par déictique «fort», tous les déictiques autres que les pronoms personnels qui sont généralement aussi classés dans la catégorie des déictiques.

${ }^{11}$ La locutrice n'a pas terminé son énoncé, mais la suite du conte permet de comprendre qu'il ne parlait plus.
} 
l'ensemble des locuteurs, soit encore d'une conservation. Dans l'état actuel des connaissances, rien ne permet de trancher entre ces trois hypothèses :

(16)

\begin{tabular}{|c|c|c|c|c|c|c|c|}
\hline $\mathbf{u}$ & bazzū-hā & & lamma & yišba $^{\Upsilon} \overline{\mathbf{u}}^{\mathbf{1 2}}$ & wun & $\operatorname{gass} \overline{\mathbf{u}}_{\text {rester }+4 \cos 3 m p l}$ & Parđ̛̣a-hā \\
\hline et & têter $+A c c 3 n$ & npl-elle & jusque & 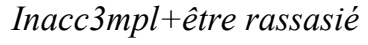 & et & rester $+A c c 3 m p l$ & près-elle \\
\hline $\begin{array}{l}\text { bi } \\
\text { avec }\end{array}$ & $\begin{array}{l}\text { l-lēel } \\
\text { art-nuit }\end{array}$ & $\begin{array}{l}\text { la } \\
\text { jusque }\end{array}$ & $\begin{array}{l}\text { s-sobh } \\
\text { art-aube }\end{array}$ & (M) & & & \\
\hline
\end{tabular}

Ils la tétaient jusqu'à ce qu'ils soient rassasiés et alors ils restaient à côté d'elle la nuit, jusqu'à l'aube.

Reste pour finir un exemple dans lequel wun permet de former une locution conjonctive avec l'adverbe négatif māšsi pour signifier « sinon », construction connue de l'arabe classique comme dialectal (avec divers adverbes de négation) :

$\begin{array}{lllllll}\text { taxabbar-hom } & \text { wen } & \text { māši, } & \text { qarạ̣ } & \text { zib } & \text { el-ḥimār, il-lēleh } \\ \text { Impmsg+s'informer-eux } & \text { et } & \text { nég } & \text { éclater }+A c c 3 m s g & \text { pénis } & \text { art-âne } & \text { art-nuit }\end{array}$

Demande-leur, sinon, putain de merde, je me casse ce soir !

\section{Valeurs sémantiques et énonciatives de la coordination avec wun}

A la lecture des exemples ci-dessus, il est déjà nettement perceptible que la relation sémantique qui s'établit entre les deux propositions reliées par wun n'est pas simplement de nature additive, qu'il ne s'agit pas de la simple description d'une suite chronologique de procès ou d'états et que la fonction de wun ne se résume pas non plus en un simple présentatif. D'autres facteurs entrent en jeu qui permettent de hiérarchiser les propositions.

On remarque tout d'abord que wun est utilisé dans des énoncés où la seconde proposition, introduite par wun, est la conséquence directe ou indirecte, le résultat d'une action ou d'un état décrit dans la première proposition, une des valeurs, rappelons-le, associée à la conjonction de coordination wa. Par ce procédé, le locuteur attire l'attention de son auditoire sur cette conséquence et rend explicite la situation résultante. Les exemples 9 et 15 en sont des illustrations, en voici quelques autres :

(18) imṭálạ̣ Calế-h l-9abríq un đā falásīs

glisser+Acc3msg sur-lui art-cruche et ce miettes

La cruche lui a glissé (des mains) et s'est retrouvée en miettes !

\begin{tabular}{|c|c|c|c|c|c|c|c|}
\hline $\begin{array}{l}\text { qafazah } \\
\text { sauter }+A c c 3 f s g\end{array}$ & $\frac{\text { wun }}{e t}$ & $\begin{array}{l}\text { đeh } \\
c e\end{array}$ & $\begin{array}{l}\text { ntakabah } \\
\text { être tomb }\end{array}$ & $+A c c 3 f s g$ & $\begin{array}{l}\text { l-a'dām } \\
\text { art-os }\end{array}$ & $\min _{d e}$ & $\begin{array}{l}\text { toqbata-hā } \\
\text { fesse de-elle }\end{array}$ \\
\hline $\begin{array}{l}\text { la?inne-hen } \\
\text { parce que-elles }\end{array}$ & $\begin{array}{l}\text { qa } \\
\text { déjà }\end{array}$ & $\begin{array}{l}\text { Cabba } \\
\text { ranger }\end{array}$ & $\begin{array}{l}\mathbf{P} \mathbf{u} \\
+A c c 3 m p l\end{array}$ & $\begin{array}{l}\text { I-lahạam } \\
\text { art-viande }\end{array}$ & $\min _{d e}$ & $\begin{array}{l}\text { tiga } \\
\text { avant }\end{array}$ & \\
\hline
\end{tabular}

Elle a sauté et voilà que les os sont tombés de ses fesses parce qu'ils y avaient déjà rangé la viande avant.

$\begin{array}{llllll}\text { fáwwar-hā } & \text { la } & \text { un } & \text { đē } & \text { mátah } \\ \text { faire bouillir }+ \text { Acc } 3 m s g-e l l e & \text { voilà } & \text { et } & \text { ce } & \text { mourir }+A c c 3 f s g\end{array}$

Il l'a fait bouillir et alors elle est morte.

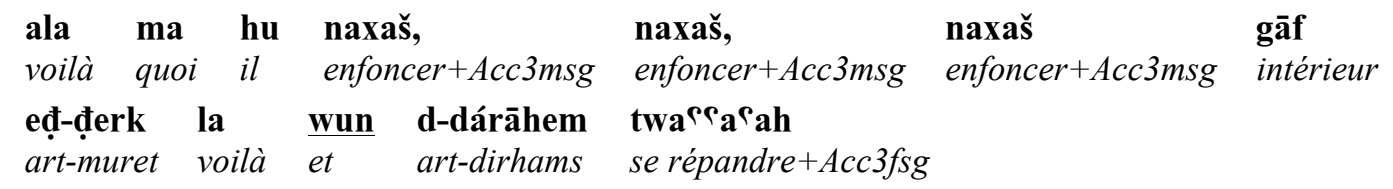

Alors il a enfoncé, enfoncé (le bras) profondément dans le muret et alors des dirhams se sont répandus.

(Comme les enfants de la première femme de son époux, la marâtre et sa fille espéraient se couvrir d'or en sautant une rivière, mais une mésaventure les attendait :)

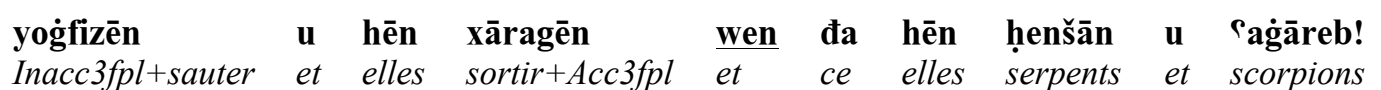

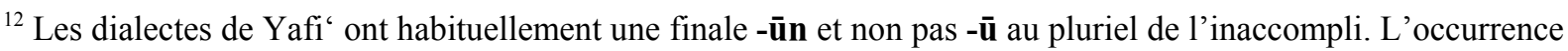
du -ū ici s'explique vraisemblablement par une influence du parler d'Aden où la locutrice a poursuivi ses études d'infirmière.
} 
Elles ont sauté, elles sont sorties et voilà qu'elles étaient couvertes de serpents et de scorpions !

Eventuellement, il faudra rechercher plus avant dans l'énoncé la proposition à laquelle se rattache celle introduite par wun, rien ne reliant sémantiquement les deux propositions directement coordonnées. Wun pourrait donner l'impression d'introduire un élément tout à fait nouveau dans le récit, mais tel n'est pas le cas, car la dépendance instaurée par wun se situe alors au niveau du texte et non plus simplement de la phrase.

Dans l'exemple ci-dessous, un père tombé enceinte abandonne sa fille par peur du qu'en dira-t-on et retourne à son village après l'accouchement, en précisant à ses voisins que son genou enflé (par lequel il a accouché) a été soigné. Et c'est parce que la fille est abandonnée, que les oiseaux, déjà mentionnés dans le conte, décident de s'occuper de son éducation. Malgré les apparences, wun ne se contente pas d'introduire un nouveau développement du conte. Comme précédemment, la seconde proposition peut être interprétée comme la conséquence d'une autre, mais qui ne la précède pas directement :

\begin{tabular}{|c|c|c|c|c|c|c|c|c|c|}
\hline $\begin{array}{l}\text { ana } \\
j e\end{array}$ & $\begin{array}{l}\mathbf{t a}^{9} \overline{\mathbf{a}} \mathbf{l e g} \mathbf{t}^{13} \\
\text { guérir }+ \text { Acc1sg }\end{array}$ & $\begin{array}{l}\text { l-ah. } \\
\text { à-lui }\end{array}$ & $\begin{array}{l}\text { hāđe } \\
c e\end{array}$ & $\begin{array}{l}\text { l-bent } \\
\text { art-fille }\end{array}$ & $\begin{array}{l}\mathbf{b a}^{\mathbf{C}} \mathbf{d} \\
\text { après }\end{array}$ & $\begin{array}{l}\text { qalīl, } \\
\text { un peu }\end{array}$ & $\begin{array}{l}\text { illa } \\
\text { voici }\end{array}$ & $\frac{\text { wun }}{e t}$ & $\begin{array}{l}\text { ț-țuyūr } \\
\text { art-oiseaux }\end{array}$ \\
\hline & $\grave{a}$-elle & aigle & & & & & & & \\
\hline
\end{tabular}

« Je me la (la tumeur) suis fait soigner. » Cette fille, peu après, voilà que les oiseaux sont venus pour elle, des aigles.

De même, dans l'ex. 24, il faut remonter à l'avant-dernière phrase, et non à la dernière précédant wun, pour trouver le lien sémantique, à savoir l'insulte qui va entraîner les pleurs de l'héroïne :

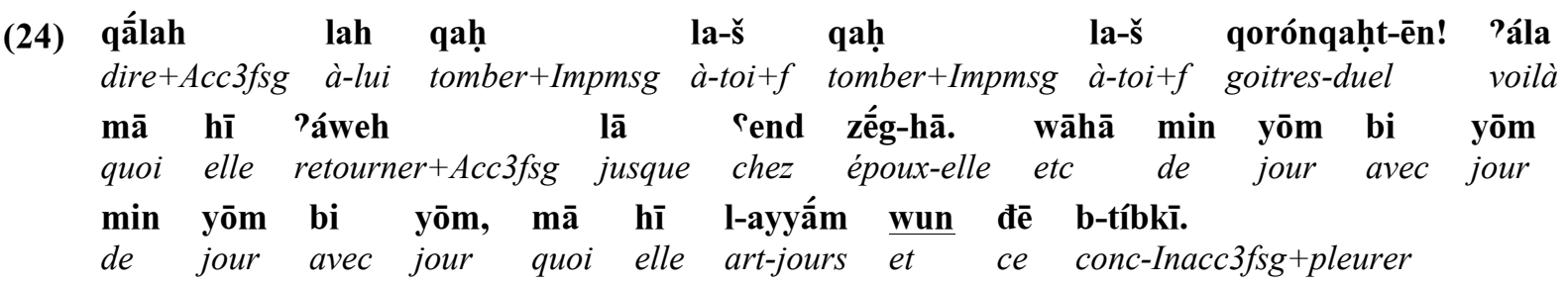

Elle lui a dit : «Que deux goitres te tombent dessus! » Voilà qu'elle est retournée chez son mari, etc., les jours ont passé jusqu'à ce qu'un jour elle se mette à pleurer.

Parfois, il est nécessaire d'avoir une connaissance du contexte culturel pour comprendre le lien sémantique de cause à effet unissant deux propositions. Ainsi l'ex. 25 fait-il référence à la coutume qui veut que la jeune mariée rende visite à ses proches après les premiers mois de mariage :

\begin{tabular}{|c|c|c|c|c|c|c|c|c|}
\hline $\begin{array}{l}\text { gess } \\
\text { rester }+ \text { Acc } 3 m s g\end{array}$ & $\begin{array}{l}\text { hu } \\
\text { il }\end{array}$ & $\begin{array}{l}\text { wēt } \\
\text { et }\end{array}$ & $\begin{array}{l}\text { el-márah } \\
\text { art-femme }\end{array}$ & $\begin{array}{l}\text { wăḥ̣d-hum } \\
\text { un-eux }\end{array}$ & $\begin{array}{ll}\text { đa. } & \text { lā } \\
c e & \text { jusque }\end{array}$ & $\begin{array}{l}\text { sána } \\
\text { année }\end{array}$ & $\frac{\mathbf{u n}}{e t}$ & $\begin{array}{l}\text { đếla } \\
\text { ceux-là }\end{array}$ \\
\hline $\begin{array}{l}\text { bi-yósș̣̄ūn } \\
\text { conc-Inacc } 3 m p l+\end{array}$ & & & her $\quad \begin{array}{l}\text { ilế-h } \\
\text { jusqu }\end{array}$ & $\begin{array}{ll} & \text { ?ahl } \\
\text {-elle } & \text { gens }\end{array}$ & $\begin{array}{l}\text { bết-ha } \\
\text { maison-elle }\end{array}$ & & & \\
\hline
\end{tabular}

Ils restèrent seuls, lui et sa femme. Après un an, voilà que ceux-là, les gens de sa famille, l'envoient chercher.

Plus rarement, ce n'est plus la conséquence qui est exprimée dans la proposition introduite par wun, mais la cause du procès énoncé dans la première proposition. Cette valeur semble être très marginale, mais on notera que, sur le plan sémantique, elle inverse simplement le rapport entre les propositions : ce n'est plus la cause qui figure dans la première proposition, mais l'effet de celle-ci, énoncée dans la seconde. Cette valeur causale, est par ailleurs une valeur fréquemment associée à la focalisation (Robert $1993: 29,38)$ :

\footnotetext{
${ }^{13}$ Cette locutrice, dont le parler est très influencé par l'arabe standard et celui d'Aden, n'a pas utilisé l'accompli en -k-.
} 


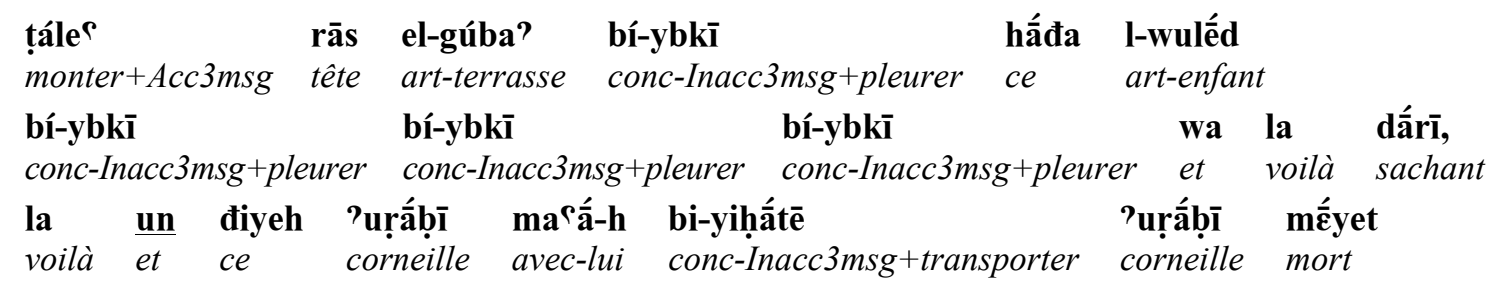

Il est monté sur la terrasse pour pleurer, cet enfant, il pleurait, pleurait et, tout à coup, il a compris car il y avait un corbeau qui transportait un corbeau mort. (Il est ensuite expliqué que c'est en regardant faire le corbeau que le héros comprend par quel moyen ressusciter son frère)

Quand wun induit un retour en arrière sur un élément du récit (marqué formellement ou non par l'adverbe qad « déjà »), l'idée de conséquence passe à l'arrière-plan et wun permet seulement d'expliquer la situation, valeur sémantique souvent associée à la focalisation (Robert 1993 : 29, 31, 38) :

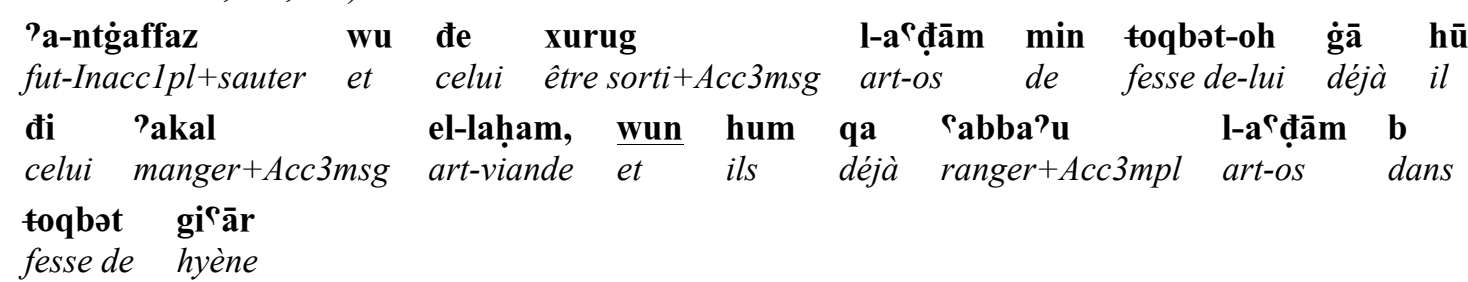

«Nous allons sauter et celui du cul duquel sortiront les os sera celui qui a mangé la viande. » $\underline{\text { C'est }}$ qu'eux avaient déjà rangé les os dans le cul de l'hyène. (Il a été spécifié plusieurs fois auparavant que c'est l'endroit où le vrai coupable a caché les os)

(la marâtre a fait avaler des œufs de serpent à sa belle-fille pour faire croire qu'elle est enceinte, mais le piège finit par être déjoué)

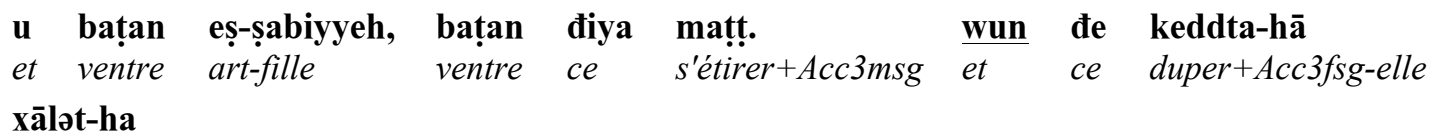

Et le ventre de la jeune fille, son ventre est redevenu plat. C'est qu'elle l'avait dupée, sa marâtre.

De même, après les verbes de mouvement et de perception, wun souligne souvent une constatation d'un état de fait, déjà mentionné auparavant ou déduit d'événements survenus antérieurement dans le conte, et sur lequel le conteur attire l'attention de son auditoire. Il ne s'agit pas dans ces cas-là de conséquences, mais comme précédemment d'une explication de la situation. Les phrases introduites par wun sont alors en général des phrases nominales, mais pas nécessairement (ex. 32) :

(la fille du roi a demandé à son père de mettre de la glu dans son trésor pour piéger un voleur)

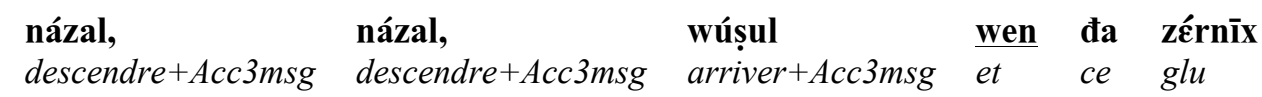

Il (le voleur) est descendu, descendu, il est arrivé, mais c'est qu'il y avait de la glu !

(en voyant l'anneau porté par le héros de l'histoire, la vieille femme qui l'avait déjà vu au doigt d'une jeune fille, devine à qui elle a affaire)

$\begin{array}{lllllllllll}\text { xáyilah } & \text { l-madwấr } & \text { la } & \text { wun } & \text { đē } & \text { ḥaqq } & \text { hấđa } & \text { đī } & \text { kấnah } & \text { hī wét-ah } \\ \text { voir }+A c c 3 f s g & \text { art-anneau } & \text { voilà } & \text { et } & c e & \dot{d} e & c e & q u i & \text { être }+A c c 3 f s g & \text { elle } & \text { et-lui }\end{array}$

Elle (la vieille femme) a vu l'anneau, mais c'est qu'il était à celle qui était avec lui (le héros) !

(la fille du roi a conseillé à son père de peindre la porte du voleur pour le repérer, mais celuici déjoue le piège en peignant toutes les portes des maisons du village)
(31) xấyel
el-`átabah wun de
mutárțaš-ah kúlla-hā
voir+Acc3msg art-porte et ce peinturluré-f tout-elle

Il (le roi) a regardé la porte, mais c'est que toutes étaient peinturlurées ! 
(pour repérer le voleur la fille du roi a conseillé à son père d'envoyer une chamelle renifleuse)

(32)

\begin{tabular}{|c|c|c|c|c|c|}
\hline $\begin{array}{l}\text { twámbas } \\
\text { s'asseoir }+A c c 3 m s g\end{array}$ & $\begin{array}{l}\text { rās } \\
\text { tête }\end{array}$ & $\begin{array}{l}\text { el-gubáa } \\
\text { art-terrasse }\end{array}$ & $\begin{array}{ll}\text { wun } & \text { đē } \\
c e\end{array}$ & $\begin{array}{l}\text { wúșuleh } \\
\text { arriver }+A c c 3 f s g\end{array}$ & $\begin{array}{l}\text { al-bákra } \\
\text { art-chamelle }\end{array}$ \\
\hline $\begin{array}{l}\text { š-šámmām-ah } \\
\text { art-renifleur-f }\end{array}$ & & $\begin{array}{l}\text { nușs } \\
\text { moitié }\end{array}$ & $\begin{array}{l}\text { ul-lếl } \\
\text { art-nuit }\end{array}$ & & \\
\hline
\end{tabular}

Il (le voleur) était assis sur la terrasse (à surveiller). Et c'est qu'elle est arrivée, la chamelle renifleuse, de nuit, au milieu de la nuit.

Wun peut aussi introduire la répétition de l'élément d'information précédent et jugé important par le locuteur. Il insiste sur le caractère effectif de la relation prédicative qui prend alors une valeur intensive ( « vraiment, effectivement»), autre valeur fréquemment associée à la focalisation (Robert 1993 : 39). Dans beaucoup des exemples de ce type, le conteur passe du dialogue au récit et donne l'impression de reprendre à son compte l'assertion, en la confirmant (pour des exemples sans ce contexte, voir ex. 1 et 7 ci-dessus) :

(33)

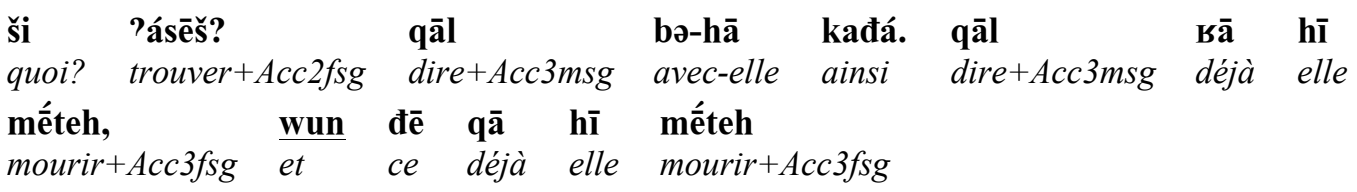

«Qu'as-tu trouvé ?», il lui a dit comme ça. Il a répondu : «Elle est déjà morte.» Et celle-ci était effectivement déjà morte.

(34)

\begin{tabular}{|c|c|c|c|c|c|c|}
\hline $\begin{array}{l}\text { illa } \\
\text { jusque }\end{array}$ & $\begin{array}{l}\text { u!̣!áh } \\
\text { Dieu }\end{array}$ & $\begin{array}{l}\text { b-ánna } \\
\text { avec-que }\end{array}$ & $\begin{array}{l}\text { áb`aš } \\
\text { Inacclsg+attraper }\end{array}$ & $\begin{array}{l}\text { el-máțhan } \\
\text { art-moulin }\end{array}$ & $\begin{array}{l}\mathbf{w} \\
\text { et }\end{array}$ & $\begin{array}{l}\text { ámțax-ok } \\
\text { Inacclsg+frapper-toi }+m\end{array}$ \\
\hline $\begin{array}{l}\text { ínteh, } \\
t u+m\end{array}$ & $\begin{array}{l}\text { ?ála } \\
\text { voilà }\end{array}$ & $\begin{array}{l}\text { đè } \\
c e\end{array}$ & $\begin{array}{l}\text { bi-yiqú́l } \\
\text { conc-Inacc } 3 m s g+\text { dire }{ }^{14}\end{array}$ & $\begin{array}{l}\text { káđa } \\
\text { ainsi }\end{array}$ & & \\
\hline
\end{tabular}

« Je jure que je vais attraper le moulin et je te frapperai, toi. » Et c'est qu'il a vraiment fait comme ça. wun :

En résumé, quatre valeurs principales sont associées aux propositions introduites par

- conséquence de la première proposition (ex. 9, 15, 18-25)

- cause de la première proposition (rare) (ex. 26)

- explicitation de la situation (ex. 2, 3, 8, 10-14, 16, 27-32)

- caractère effectif du procès ou de l'état (intensif) (ex. 1, 7, 33, 34)

Sur le plan énonciatif, quelle que soit la valeur sémantique des énoncés avec wun, celuici met toujours en valeur d'une manière ou d'une autre la proposition qu'il introduit en attirant l'attention des auditeurs sur l'importance des faits et procès qui y sont énoncés. L'information ainsi véhiculée correspond souvent à un moment clé du récit et constitue le rhème d'un énoncé. On a également souligné à plusieurs reprises, les affinités entre des valeurs apportées par wun et celles liées à la focalisation. Mais pour confirmer une éventuelle appartenance de wun à cette opération énonciative, de simples rapprochements sémantiques ne suffisent pas et nous allons maintenant tenter de dégager les traits syntaxiques communs à la focalisation et au fonctionnement de wun.

\section{Conclusion}

Certains linguistes, et c'est la position adoptée ici, s'accordent pour définir la focalisation comme un type d'assertion complexe, comme « un mode particulier d'identification » (Robert 1993 : 25), faisant intervenir la désignation, et donc l'existence, de l'objet de l'assertion (le rhème), l'effectivité de la relation prédicative et la préconstruction de cette relation. Pour Robert (1993 : 25), la focalisation est une assertion qui «a pour objet la désignation qualitative d'un élément dont l'existence est préconstruite », et dont les emplois « peuvent se ramener à trois grands cas : identification, explication, exclamation intensive. » Caron (à

\footnotetext{
${ }^{14} \mathbf{q} \mathbf{a} \mathbf{l}$, comme dans beaucoup de dialectes, a aussi le sens de "faire".
} 
paraître) en propose la définition syntaxique et sémantique suivante : « La focalisation est l'imbrication, la présentation simultanée, dans un seul énoncé, d'un élément identifié (avec ou sans contraste) avec la place vide d'un terme d'une relation prédicative. L'assertion porte sur l'identification, alors que la relation prédicative est préconstruite ». Préconstruction s'entend au sens que lui accorde Culioli c'est-à-dire comme « une relation prédicative posée comme validée par rapport à un repère-origine externe à l'énoncé en cours. 》 (ibid.).

Le marqueur wun de l'arabe de Yafi' répond, nous semble-t-il, à ces critères :

- L'énoncé dans lequel figure wun constitue bien une unité syntaxique, en l'occurrence une phrase complexe à deux termes, c'est-à-dire à deux propositions, dont la seconde constitue l'élément focalisé.

- L'identification, l'existence de l'objet de l'assertion, c'est-à-dire le rhème, est formellement marquée par l'utilisation de déictiques (présentatifs et démonstratif) dans la proposition focalisée : on rappellera l'origine même du -n final, une particule dont l'une des fonctions est d'être un présentatif, le recours quasi systématique à un démonstratif à base đqui suit directement wun, enfin le renforcement possible par le présentatif (il)la " voilà ». Autant de morphèmes qui permettent d'affirmer l'existence du rhème, de le désigner. Avec wun, ce n'est pas seulement l'un des termes tels que le sujet ou l'objet qui constitue la "place vide» que tout marqueur de focalisation doit identifier en le focalisant, mais l'ensemble de la proposition dépendante dans une phrase complexe.

Par ailleurs, on notera que le -n final de wun semble être en train de perdre, dans le processus diachronique d'agglutination, de grammaticalisation, sa valeur déictique, celle justement qui permet de désigner l'objet de l'assertion. Il est probable en effet que le nombre très élevé d'occurrences du démonstratif ou du présentatif au sein des propositions focalisées soit le signe que la langue est en train de surmonter cette évolution en rendant obligatoire le premier d'entre eux. Les phrases sans déictique pourraient ne plus être que des scories. Ce n'est peut-être pas un hasard si, lorsque wun n'est pas agglutiné mais apparaît sous sa forme pleine, wu suivi de 'in(na), il n'a été relevé aucun exemple avec un élément déictique supplémentaire. Mais il convient de rester prudent sur les implications diachroniques de cette remarque vu le petit nombre d'exemples de ce type relevé dans le corpus. Danon-Boileau (1992 : 22) a souligné le « lien entre deixis et promotion focale. » L'arabe de Yafi“ en est une illustration supplémentaire.

- La préconstruction se traduit par la présence de morphèmes instaurant une dépendance syntaxique, wun étant composé d'une conjonction de coordination wu, pouvant avoir valeur subordonnante, et d'une conjonction de subordination ?in(na). Wun permet d'articuler « l'énoncé focalisé au contexte variable qui lui sert de repère » (Robert 1993 : 25), à savoir la proposition qui précède ou tout autre contexte énoncé antérieurement et qui constitue le repère-origine externe.

- L'effectivité de la relation prédicative : celle-ci est posée comme validée et fait partie du domaine de l'assertion (affirmative ou négative); elle n'est jamais associée à une modalité épistémique ou déontique, ne peut être mise en doute, à tel point que la proposition focalisée peut s'interpréter comme une insistance sur ce caractère effectif ( $c f$. les traductions par « vraiment », « effectivement »).

- Pour ce qui est du caractère explicatif, on a vu que la construction avec wun est très souvent associée à une valeur d'explication de la situation, et qu'elle peut même parfois exprimer la cause du procès énoncé dans la première proposition.

- Quant à la valeur de conséquence dégagée pour certains énoncés, si elle ne figure pas dans l'inventaire des valeurs associées à la focalisation par Robert (1993), elle ne nous semble pas devoir remettre en cause l'interprétation qui a été proposée. D'une part parce que toutes les autres caractéristiques syntaxico-sémantiques de la focalisation sont présentes et, d'autre part, parce que le lien de cause à effet instauré entre les deux propositions est simplement inversé. Il semble que la valeur saillante des énoncés où s'expriment cause ou conséquence soit plutôt le rapport de cause à effet lui-même, que le sens dans lequel il est énoncé. 
Wun n'appartient donc pas plus à la catégorie grammaticale des conjonctions de coordination, qu'à celle des conjonctions de subordination ou à la deixis. Il y participe certes de par son origine, mais son fonctionnement actuel est bien celui d'un morphème de focalisation.

On pourrait se demander également si wun n'a pas conservé le rôle de morphème de topicalisation (appelé thématisation dans Fernandez 1994) dévolu à Pin(na). Si l'on admet que le topique figure nécessairement en tête d'énoncé et ne constitue pas une unité syntaxique avec celui-ci mais en est détaché ( $c f$. Caron à paraître), alors il ne fait pas de doute que l'arabe de Yafi' n'en a pas conservé la trace.

Enfin, sur le plan rhétorique, on a vu que wun apparaît à des moments clés du récit. On pourrait dire, pour reprendre une terminologie chère à David Cohen, qu'il constitue un procédé de retardement. Il permet en effet de maintenir le public en haleine, d'attirer son attention sur une action importante, et de ménager un certain effet de surprise ( $c f$. ex. 3) ou de soudaineté, phénomènes dont rendent compte les traductions proposées par Piamenta $(c f . \S 1)$ et que l'arabe de Yafi' partage avec d'autres variétés d'arabe.

Au cours leur histoire, comme dans leurs fonctionnements synchroniques, les langues ne connaissent pas de frontière perméable entre les catégories grammaticales, la transcatégorialité étant, au contraire, un des moteurs des évolutions. La particule énonciative de focalisation de l'arabe de Yafi' en est une modeste illustration.

\section{Abréviations}

$\begin{array}{llll}\text { Acc } & \text { accompli } & \text { Inacc } & \text { inaccompli } \\ \text { art } & \text { article } & \mathrm{m} & \text { masculin } \\ \text { conc } & \text { concomitant } & \mathrm{nég} & \text { négation } \\ \mathrm{f} & \text { féminin } & \mathrm{pl} & \text { pluriel } \\ \text { fut } & \text { futur } & \mathrm{sg} & \text { singulier } \\ \text { Imp } & \text { impératif } & & \end{array}$

\section{Références citées}

Blachère, Régis \& M. Gaudefroy-Demombynes, 1975, Grammaire de l'arabe classique, Paris, Maisonneuve et Larose.

Caron, Bernard, à paraître, Assertion et préconstruction dans les langues africaines. Problèmes de topicalisation et de focalisation, Préconstruit, focalisation et topicalisation dans les langues africaines, B. Caron éd., Louvain, Peeters.

Danon-Boileau, Laurent, 1992, Présentation, La deixis. Colloque en Sorbonne 8-9 juin 1990, M. A. Morel \& L. Danon-Boileau éds., Paris, PUF : 11-25.

Fernandez, Jocelyne, 1994, Les particules énonciatives, Paris, PUF.

Landberg, Carlo, de, 1901, Etudes sur les dialectes de l'Arabie Méridionale. Premier volume : Haḍamaût, Leide, Brill.

Landberg, Carlo, de, 1905, Etudes sur les dialectes de l'Arabie Méridionale. Deuxième volume: Datînah. Première partie. Textes et traduction, Leide, Brill.

Piamenta, Moshe, 1990-1991, Dictionary of Post-Classical Yemeni Arabic, Leiden, Brill.

Robert, Stéphane, 1993, "Structure et sémantique de la focalisation”, BSLP, LVIII, 1 : 25-47.

Rossi, Ettore, 1939, L'Arabo parlato a San 'â'. Grammatica - Testi - Lessico, Roma, Istituto per l'Oriente.

Vanhove, Martine, 1995, "Notes on the Arabic Dialectal Area of Yafi'.(Yemen)", Proceedings of the Seminar for Arabian Studies (Oxford, July 1994), Vol 25, 141-152.

Watson, Janet, 1993, A Syntax of San 'ani Arabic, Wiesbaden, Harrassowitz. 